\title{
Linfoma folicular duodenal primario: reporte de caso y revisión de la literatura
}

\section{Primary duodenal follicular lymphoma: Case report and literature review}

\author{
Lázaro Antonio Arango-Molano, ${ }^{1}$ [D Andrés Sánchez-Gil, ${ }^{2}$ (D) Ileana Rocío Bautista-Parada. ${ }^{*}$ (1)
}

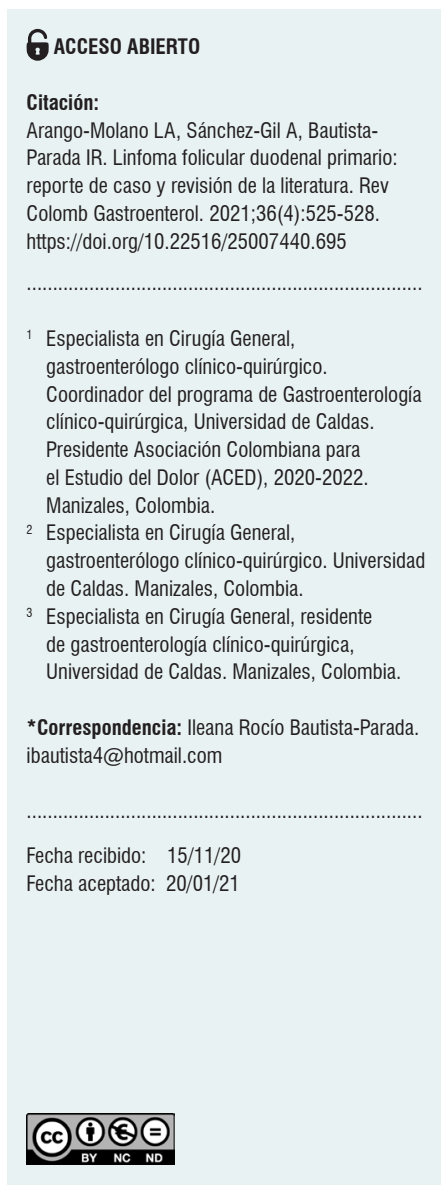

\section{Resumen}

Los linfomas primarios del tracto gastrointestinal son infrecuentes; sin embargo, son la presentación extranodal más común de los linfomas no Hodgkin. El $30 \%$ de los linfomas no Hodgkin corresponde a linfomas foliculares y, a su vez, cerca del $10 \%$ de los linfomas foliculares se origina en el tracto gastrointestinal. Se han descrito factores de riesgo para el desarrollo de linfomas gastrointestinales como infección por Helicobacter pylori, inmunosupresión posterior a trasplante de órganos sólidos, enfermedad inflamatoria intestinal e infección por virus de la inmunodeficiencia humana $(\mathrm{VIH})$. El linfoma duodenal folicular se reconoció como una variante del linfoma folicular en 2016 según la clasificación de la Organización Mundial de la Salud (OMS), al considerar que se trata de una condición con características biológicas y clínicas particulares. Su diagnóstico suele ser incidental o se pueden presentar síntomas leves e inespecíficos. El grado histológico suele ser bajo y el curso clínico, benigno; por lo que en gran parte de los casos se ha adoptado el manejo expectante como una opción. Otras terapias con similar efectividad son la radioterapia, el uso de rituximab y la inmunoquimioterapia. No existe a la fecha suficiente evidencia para generar un protocolo único de manejo para esta patología.

\section{Palabras clave}

Linfoma folicular, linfoma duodenal, linfoma no Hodgkin extraganglionar.

\begin{abstract}
Primary gastric lymphomas are rare diseases; however, they are the most common extranodal presentation of non-Hodgkin lymphomas. $30 \%$ of non-Hodgkin lymphomas correspond to follicular lymphomas and at the same time, nearly $10 \%$ of follicular lymphomas are produced in the gastrointestinal tract. Risk factors for gastric lymphomas such as Helicobacter pylori infection, immunosuppression after solid organ transplantation, inflammatory bowel disease, and human immunodeficiency virus (HIV) infection were described. Follicular duodenal lymphoma was recognized as a variant of follicular lymphoma in 2016 according to the World Health Organization (WHO) classification, considering that it is a condition with special biological and clinical characteristics. Its diagnosis is usually incidental or mild and nonspecific symptoms may occur. The histological grade is usually low, and the clinical course is benign; Therefore, in most cases, expectant treatment has been adopted as an option. Other therapies with similar effectiveness are radiotherapy, the use of rituximab, and immunochemotherapy. There is not enough evidence to date to generate a single management protocol for this pathology.
\end{abstract}

\section{Keywords}

Folicular lymphoma, Duodenal lymphoma, Extraganglionar non-Hodgkin's lymphoma.

\section{INTRODUCCIÓN}

El tracto gastrointestinal es un componente importante del sistema inmune y en él existe tejido linfoide en cantidades y tipos variables. El esófago y estómago poseen poco tejido linfoide mucoso, a diferencia del intestino, en el que es abundante, de manera predominante en la mucosa y submucosa; este tejido es conocido como tejido linfoide aso- 
ciado a mucosa (MALT). La proliferación monoclonal del MALT en respuesta a la estimulación antigénica crónica o inflamación puede llevar al desarrollo de varias formas de linfoma gastrointestinal ${ }^{(1)}$. Los linfomas primarios del tracto gastrointestinal son raros; sin embargo, corresponden a la ubicación extranodal más frecuente en la que se desarrollan los linfomas no Hodgkin (LNH), pues corresponden al $30 \%$ de los $\operatorname{casos}^{(2,3)}$.

El linfoma duodenal folicular (LDF) es una variante rara del linfoma folicular (LF), reconocida de manera reciente como subtipo de este último en la clasificación de la Organización Mundial de la Salud (OMS) debido a su naturaleza distintiva ${ }^{(4)}$.

\section{PRESENTACIÓN DEL CASO}

Se trata de una paciente femenina de 42 años sin antecedentes patológicos, quien consultó en diciembre de 2013 por síntomas dispépticos de larga data y con estudio endoscópico que informaba una gastropatía crónica atrófica, papila mayor de aspecto adenomatoso y lesión elevada en la tercera porción del duodeno. Se realizó una ultrasonografía endoscópica (USE) que evidenció el engrosamiento mucoso en la tercera porción del duodeno sin compromiso de otras capas; se realizó la toma de biopsia cuyo informe sugirió un proceso linfomatoso. El estudio de inmunohistoquímica confirmó LF grado 1 y la tomografía toracoabdominal realizada en ese momento no mostró compromiso ganglionar alguno.

La paciente abandonó los controles y reconsultó en febrero de 2020, se realizó una nueva esofagogastroduodenoscopia en la que se identificó un bulbo duodenal normal y segunda porción del duodeno con mucosa nodular, blanquecina y friable. Se tomaron biopsias que mostraron LF de bajo grado, e inmunohistoquímica con marcadores MUM negativos, kappa, lambda y CD3 positivos en linfocitos acompañantes compatibles con LDF de bajo grado (Figura 1). Se realizó una tomografía de control en la que no se encontró compromiso ganglionar o de otros órganos. Actualmente, la paciente se encuentra en manejo y seguimiento por oncología clínica.

\section{DISCUSIÓN}

El LF es uno de los linfomas de células B de bajo grado más comunes, corresponde al $30 \%$ de todos los LNH en países occidentales ${ }^{(4)}$. El tracto gastrointestinal es la localización más frecuente de las presentaciones extranodales y cerca del $10 \%$ de todos los LF es de origen gastrointestinal $^{(5)}$. Se han identificado varios factores de riesgo para el desarrollo de linfomas gastrointestinales, como la infección por Helicobacter pylori, inmunosupresión posterior a

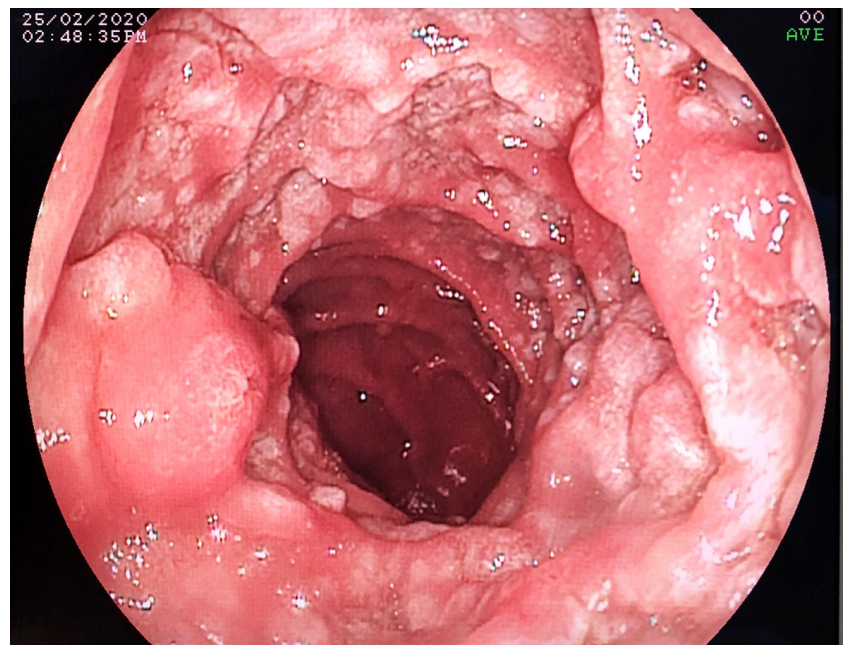

Figura 1. Hallazgos endoscópicos: lesiones nodulares de aspecto granular en la segunda porción del duodeno.

trasplante de órganos sólidos, enfermedad inflamatoria intestinal (EII) e infección por virus de inmunodeficiencia humana $(\mathrm{VIH})^{(3)}$. La alteración genética distintiva de los LF es la translocación $t(14 ; 18)$ (q32; q21) de los genes de la cadena pesada de la inmunoglobulina (Ig). Además, se han descrito ciertas similitudes en el perfil genético con el linfoma MALT, estas similitudes están asociadas con la estimulación antigénica en inflamación crónica, y se sospecha que el LDF puede seguir un curso similar al linfoma MALT, que se origina sobre inflamación preexistente ${ }^{(6)}$.

El primer caso de LFD se reportó en 1997 e históricamente el LF gastrointestinal se estableció como enfermedad en la década del 2000. A medida que se fue reconociendo como entidad, se reportaron series de casos y, como resultado, se estableció que el linfoma intestinal folicular primario corresponde a una variante de la clasificación de los linfomas foliculares según la clasificación realizada por la OMS; dentro de esta también se reconoce al linfoma duodenal como una entidad de naturaleza específica, que aunque tiene las características de un LF localizado de bajo grado, es distinta de otros LF gastrointestinales ${ }^{(7)}$. El duodeno (65\%), íleon y yeyuno $(20 \%)$ son los sitios del tracto gastrointestinal más frecuentemente afectados por el LF, aunque también se han reportado casos en el colon, recto y estómago ${ }^{(5)}$.

El LDF se presenta de manera predominante en adultos de edad media con distribución similar entre hombres y mujeres $^{(8)}$. La mayoría de los pacientes es asintomática $y$, por tanto, el diagnóstico suele ser incidental, generalmente durante un estudio endoscópico indicado por un motivo no relacionado. Cuando se presentan, las manifestaciones clínicas suelen ser síntomas gastrointestinales superiores como dolor, incomodidad abdominal, vómito y, menos frecuentemente, sangrado gastrointestinal ${ }^{(7,9)}$. Los hallaz- 
gos endoscópicos son en la mayoría de los casos descritos como lesiones blanquecinas nodulares únicas o múltiples, de aspecto granular y no de apariencia submucosa; en otros casos se mencionan como pequeños nódulos polipoides de entre 1 y $5 \mathrm{~mm}$. De manera menos frecuente se presentan como erosiones o úlceras, en este caso es importante tomar muestras del área circundante para un mayor rendimiento diagnóstico. Algunos autores han reportado la identificación de manchas blanquecinas opacas, vellosidades aumentadas de tamaño y un patrón de dilatación vascular en las vellosidades como hallazgos característicos de esta patología al emplear coloración electrónica. La coloración blanquecina de las lesiones intestinales se atribuye a la infiltración de células linfomatosas en las vellosidades ${ }^{(8-10)}$. La mayoría de los casos se presenta como una patología localizada, pero cuando se completa el estudio del intestino delgado, hasta el $85 \%$ de los pacientes con LFD tiene compromiso yeyunal o ileal ${ }^{(9)}$.

En el estudio histológico, los folículos neoplásicos son similares a los identificados en la enfermedad nodal, compuestos de una población uniforme de centrocitos, usualmente con hendiduras nucleares y algunos centroblastos. El grado histológico se define de acuerdo con el número de centroblastos por 40 campos de alto poder (CAP): grado 1 : 5 o menos centroblastos por CAP, grado 2: 6-15 centroblastos por CAP, y grado 3: más de 15 centroblastos por CAP. El grado 3 se subdivide en $3 a$ : centrocitos aún presentes y $3 \mathrm{~b}$ : sábanas de centroblastos ${ }^{(11,12)}$. Más del $95 \%$ de los casos de LFD son grado 1 a 2 (bajo grado) ${ }^{(4,7)}$. Un hallazgo histológico típico es el "patrón duodenal" en el que las células dendríticas foliculares se ubican en la periferia de los folículos neoplásicos, a diferencia de la enfermedad nodal, en la que estas forman una malla densa dentro de los folículos ${ }^{(5)}$.

Las células linfomatosas muestran un inmunofenotipo similar al de la enfermedad nodal de bajo grado, con expresión de los antígenos CD20 y CD10, y linfoma de células B tipo 2 (BCL-2) y tipo 6 (BCL-6); y la tasa de proliferación Ki-67 es baja. A diferencia de la enfermedad sistémica, el LDF no expresa activación inducida por citidina desaminasa (AID) y expresa positividad para inmunoglobulina A (IgA), BACH2 y el antígeno $\mathrm{CD} 27^{(2,5,8)}$.

Una vez se tiene la confirmación histológica, el proceso de estadificación incluye un examen físico completo, estudio tomográfico de cuello y toracoabdominal, así como estudios de química sanguínea (hemograma, lactato deshidrogenasa $[\mathrm{LDH}]$, función renal, enzimas hepáticas) y aspirado de médula ósea. Se puede considerar la realización de tomografía por emisión de positrones (PET scan), sobre todo si se estima conveniente el manejo con radioterapia y completar el estudio con enteroscopia de doble balón para evaluar la totalidad del intestino delgado. Para deter- minar si se trata de un linfoma gastrointestinal primario se han propuesto algunos criterios: ausencia de adenomegalias palpables, ausencia de adenomegalias mediastinales, recuento diferencial leucocitario normal, enfermedad limitada al intestino y ganglios adyacentes, sin compromiso de hígado o bazo ${ }^{(13,14)}$.

Según el compromiso único o múltiple en el tracto gastrointestinal, grado de infiltración de la pared intestinal, compromiso ganglionar secundario, de órganos adyacentes o a distancia, se puede hacer una estadificación de la enfermedad de I a IV (Clasificación de Lugano) (Tabla 1) ${ }^{(15)}$.

Tabla 1. Clasificación de Lugano para linfomas extranodales ${ }^{(15)}$
Clasificación

Estadio I

Estadio IE

Estadio II

Estadio III

Estadio IV

\section{Características}

Compromiso de un solo órgano linfático

Compromiso extralinfático único en ausencia de compromiso ganglionar

Compromiso de dos o más zonas ganglionares al mismo lado del diafragma

Compromiso ganglionar a ambos lados del diafragma Compromiso diseminado

El LFD se considera una patología indolora con muy buen pronóstico y tasas de supervivencia promedio de más de 12 años. Teniendo en cuenta el curso usualmente asintomático de esta condición y la poca frecuencia de progresión o transformación histológica, por una parte, se ha propuesto el manejo expectante como opción válida para estos pacientes, pues ha demostrado ser una estrategia igualmente efectiva incluso con remisiones espontáneas en algunos casos. Por otra parte, se ha reportado que los pacientes tratados con radioterapia como terapia inicial tienen tasas de supervivencia a 10 años hasta del $80 \%$; por tanto, es posible que en algunos casos esta terapia sea curativa, teniendo en cuenta que es poco probable una recaída después de este tiempo. Cuando existe recaída, la supervivencia disminuye al $22 \%$ a 10 años. La quimioterapia adyuvante no ha demostrado beneficio adicional después de la radioterapia ${ }^{(8,16)}$. La monoterapia con rituximab (anticuerpo monoclonal humanizado anti-CD20) ha sido una opción de tratamiento en LF, y su efectividad se ha demostrado con remisiones prolongadas, aun en recaídas, o como segunda línea de tratamiento. Se ha comparado también el manejo expectante con la inmunoquimioterapia, en seguimientos hasta de 149 meses y se encontraron resultados similares ${ }^{(9)}$. Todas estas podrían ser entonces estrategias válidas de tratamiento, aunque no hay hasta el momento consenso sobre la mejor opción de manejo para el LDF. 


\section{CONCLUSIONES}

El LDF es una condición rara, con presentación clínica y comportamiento biológico particulares, por lo que ha sido reconocido de manera reciente como variante del LF según la clasificación de la OMS. Suele ser asintomático y de manera infrecuente presenta transformación histológica $o$ progresión a enfermedad nodal. No se cuenta con suficiente evidencia en la literatura para establecer un protocolo de manejo; sin embargo, teniendo en cuenta su particular comportamiento benigno, el manejo expectante puede considerarse en gran parte de los casos; otras opciones terapéuticas que han demostrado alta efectividad incluyen el uso de radioterapia, rituximab e inmunoquimioterapia.

\section{REFERENCIAS}

1. Manning MA, Somwaru AS, Mehrotra AK, Levine MS. Gastrointestinal Lymphoma: Radiologic-Pathologic Correlation. Radiol Clin North Am. 2016;54(4):765-84. https://doi.org/10.1016/j.rcl.2016.03.007

2. Weindorf SC, Smith LB, Owens SR. Update on Gastrointestinal Lymphomas. Arch Pathol Lab Med. 2018;142(11):1347-1351. https://doi.org/10.5858/arpa.2018-0275-RA

3. Ghai S, Pattison J, Ghai S, O’Malley ME, Khalili K, Stephens M. Primary gastrointestinal lymphoma: spectrum of imaging findings with pathologic correlation. Radiographics. 2007;27(5):1371-88. https://doi.org/10.1148/rg.275065151

4. Swerdlow SH, Campo E, Pileri SA, Harris NL, Stein H, Siebert R, Advani R, Ghielmini M, Salles GA, Zelenetz AD, Jaffe ES. The 2016 revision of the World Health Organization classification of lymphoid neoplasms. Blood. 2016;127(20):2375-90. https://doi.org/10.1182/blood-2016-01-643569

5. Takata K, Miyata-Takata T, Sato Y, Iwamuro M, Okada H, Tari A, Yoshino T. Gastrointestinal follicular lymphoma: Current knowledge and future challenges. Pathol Int. 2018;68(1):1-6. https://doi.org/10.1111/pin.12621

6. Yoshino T, Takata K, Tanaka T, Sato Y, Tari A, Okada $\mathrm{H}$. Recent progress in follicular lymphoma in Japan and characteristics of the duodenal type. Pathol Int. 2018;68(12):665-676. https://doi.org/10.1111/pin.12733

7. Iwamuro M, Kondo E, Takata K, Yoshino T, Okada H. Diagnosis of follicular lymphoma of the gastrointestinal tract: A better initial diagnostic workup. World J Gastroenterol. 2016;22(4):1674-83. https://doi.org/10.3748/wjg.v22.i4.1674

8. Marks E, Shi Y. Duodenal-Type Follicular Lymphoma: A Clinicopathologic Review. Arch Pathol Lab Med. 2018;142(4):542-547. https://doi.org/10.5858/arpa.2016-0519-RS
9. Duffles Amarante G, Collins G, Rocha V. What do we know about duodenal-type follicular lymphoma? From pathological definition to treatment options. $\mathrm{Br} \mathrm{J}$ Haematol. 2020;188(6):831-837. https://doi.org/10.1111/bjh.16348

10. Iwamuro M, Okuda M, Yumoto E, Suzuki S, Shirakawa A, Takata K, Yoshino T, Okada H, Yamamoto K. Magnifying endoscopy for intestinal follicular lymphoma is helpful for prompt diagnosis. Gut Liver. 2013;7(2):258-61. https://doi.org/10.5009/gnl.2013.7.2.258

11. Campo E, Swerdlow SH, Harris NL, Pileri S, Stein H, Jaffe ES. The 2008 WHO classification of lymphoid neoplasms and beyond: evolving concepts and practical applications. Blood. 2011;117(19):5019-32. https://doi.org/10.1182/blood-2011-01-293050

12. Freedman A. Follicular lymphoma: 2018 update on diagnosis and management. Am J Hematol. 2018;93(2):296305. https://doi.org/10.1002/ajh.24937

13. Castro D, Beltrán B, Quiñones MDP, Palomino E, Cotrina E, Alva E, Medina JB, Yábar A. Linfoma folicular tipo duodenal: reporte de caso y revisión de la literatura. Rev Gastroenterol Peru. 2019;39(3):276-9.

14. Fujishima F, Katsushima H, Fukuhara N, Konosu-Fukaya S, Nakamura Y, Sasano H, Ichinohasama R. Incidence Rate, Subtype Frequency, and Occurrence Site of Malignant Lymphoma in the Gastrointestinal Tract: PopulationBased Analysis in Miyagi, Japan. Tohoku J Exp Med. 2018;245(3):159-165. https://doi.org/10.1620/tjem.245.159

15. Boot $\mathrm{H}$. Diagnosis and staging in gastrointestinal lymphoma. Best Pract Res Clin Gastroenterol. 2010;24(1):3-12. https://doi.org/10.1016/j.bpg.2009.12.003

16. Lee H, Oh D, Yang K, Ko YH, Ahn YC, Kim WS, Kim SJ. Radiation Therapy Outcome and Clinical Features of Duodenal-Type Follicular Lymphoma. Cancer Res Treat. 2019;51(2):547-555. https://doi.org/10.4143/crt.2018.190 